\title{
Design of novel starch-based Pickering emulsions as platforms for skin photoprotection
}

\author{
J. Marto a , L.F. Gouveia a ${ }^{\text {, L. Gonçalves }}{ }^{\text {a }}$, B.G. Chiari-Andréo ${ }^{\text {b }}$, V. Isaac ${ }^{\text {b }}$, P. Pinto ${ }^{\text {a }}$, E. Oliveira ${ }^{\text {, }}$, \\ A.J. Almeida a , H.M. Ribeiro ${ }^{\mathrm{a}, *}$ \\ a Research Institute for Medicines and Pharmaceutical Sciences (iMed.ULisboa), Faculty of Pharmacy, University of Lisbon, Portugal \\ b UNESP - Univ. Estadual Paulista, Faculdade de Ciências Farmacêuticas, Araraquara, Brazil \\ c Laboratórios Atral S.A., Lisbon, Portugal
}

\section{A R T I C L E I N F O}

\section{Article history:}

Received 15 October 2015

Received in revised form 13 June 2016

Accepted 14 June 2016

Available online 16 June 2016

\section{Keywords:}

Photoprotection

Physical UV filters

Broad-spectrum Pickering sunscreen

Water resistance

Natural ingredients

\begin{abstract}
A B S T R A C T
Green coffee oil and modified starch were recently found to have an enhanced protection effect against UV radiation. Therefore, this work aimed to develop an innovative sunscreen formulation based on Pickering emulsions concept, i.e., surfactant-free emulsions stabilized by physical UV filters associated natural oils as a key strategy for prevention against UV-induced skin damage.

The Pickering emulsions of different compositions were characterized in terms of $\mathrm{pH}$, mechanical, physical and microbiological stability by a thorough pharmaceutical control. In addition, the sun protection factor (SPF) as well as the in vitro and in vivo biological properties of the final formulations, including Episkin ${ }^{\circledR}$, HRIPT and sunscreen water resistance.

Formulation studies demonstrated the addition of physical UV filters was beneficial, leading to the inclusion of $\mathrm{ZnO}$ and $\mathrm{TiO}_{2}$ to ensure a high SPF against UVA and UVB, respectively. Although starch particles presented no intrinsic photoprotection properties, they proved to be a SPF promoter by a synergistic effect. Green coffee oil was the selected natural oil due to the highest SPF, when compared to other natural oils tested. Besides the excellent sunscreen activity confirmed by in vitro and in vivo results, the final formulations proved to be also suitable for topical use according to the rheological assessment and stability throughout the study period ( 3 months).

In conclusion, the combination of three multifunctional solid particles and green coffee oil, contributed to achieve a stable and effective innovative sunscreen with a wide range of UV radiation protection.
\end{abstract}

(c) 2016 Elsevier B.V. All rights reserved.

\section{Introduction}

Over the last decades, skin cancer has shown to be one of the most common types of cancers worldwide with an increasing incidence, mainly the non-melanoma skin cancer (NMSC) [1,2]. Ultraviolet radiation (UVR) from sun exposure is the main aetiological agent for skin cancer $[3,4]$. Avoiding sun exposure, covering the skin and applying sunscreens with a high degree of protection are the main strategies recommended to prevent UV-induced cell damage. The UV filters of sunscreens can be divided in two groups: a) organic or chemical filters which absorb UVR and are "not visible" on skin surface, being more unstable and reactive; and b) inorganic or physical filters, which reflect UVR. However, the main limitations of the physical filters are related to their poor spreadability and less cosmetic appearance on the skin [5]. Therefore, the research of new UV filters, or at least, an alternative to reduce their concentration without affecting the total photoprotection of

\footnotetext{
* Corresponding author at: Research Institute for Medicines (iMed.ULisboa), Faculty of Pharmacy, Universidade de Lisboa, Av. Professor Gama Pinto, 1649-003 Lisboa, Portugal.

E-mail address: helena.ribeiro@ff.ul.pt (H.M. Ribeiro).
}

the sunscreen product should be promoted to obtain effective, greener and safer sunscreen formulations.

The efficacy of sunscreen products has been recognized as an important public health issue and is usually expressed by the sun protection factor (SPF), which is defined as the UV energy required to produce a minimal erythema dose (MED) on protected skin, divided by the UV energy dosage required to produce a MED on unprotected skin [6]. Thus, the determination of SPF represents the first approach to evaluate the ability of sunscreens to prevent skin damage by UVR [7].

The photoprotection afforded by topical sunscreens against solar UVR exposure can be determined in vitro and in vivo, ideally in human volunteers. In vivo SPF determination has been used for many years. Although useful and precise, it is also a time consuming, complex and expensive process, particularly when the determination of the protection against long wavelength (UVA) is also required [8]. Thus, in vitro determination can be an alternative and faster way to obtain information about the photoprotective activity of UV filters. The in vitro SPF can be calculated by UV spectrophotometric technique employing a very simple mathematical equation developed by Mansur et al. [9] or using a specific UV spectrophotometer covering both UVB and UVA, 
optimized for the determination of SPF on sunscreens and cosmetic products.

Pickering emulsions are surfactant-free liquid or semi-solid systems stabilized by solid particles (SP). This type of emulsion has important advantages over the classic surfactant-based emulsions, as a higher resistance to coalescence due to an improved stability (especially with a high internal phase ratio) as well as a higher tolerability, and easy production of stable large droplets up to the millimetre size [10]. In Pickering emulsions, the solid stabilizing particles are necessarily smaller than emulsion droplets.

Stabilization of emulsion droplets takes place by means of adsorption of SP at the surface of emulsion droplets. It can be assumed that the stability of Pickering emulsion is a function of particle concentration, $\mathrm{pH}$ and ionic strength [11]. This mechanism of adsorption is rather different from that of surfactants since the SP do not need to be made of amphiphilic molecules. Partial wetting of the SP surface by water and oil is the origin of the strong anchoring of these particles at the oil-water interface. In fact, only few natural and biocompatible materials are available for the successful stabilization of these emulsions since strict requirements, including the insolubility in both fluid phases and intermediate wettability, need to be met. The principal parameters of SP affecting their physicochemical properties include shape, size, surface characteristics and inner structure [11].

Therefore, several types of SP can be used for the stabilization of Pickering emulsions. Starch, a well-known pharmaceutical excipient, shows strong affinity for the oil-water interface resulting in stable emulsions [12]. In addition, starch has been chemically modified to improve many aspects of pharmaceutical emulsions, such as: spreadability, oil absorption, water repellence, and heat tolerance [13]. Some UV filters, as titanium dioxide $\left(\mathrm{TiO}_{2}\right)$ and zinc oxide $(\mathrm{ZnO})$, can be also used as $\mathrm{SP}$ for the stabilization of these emulsions [14]. $\mathrm{TiO}_{2}$ has been incorporated in sunscreen formulations for $>25$ years, and it has been regarded as safe and effective, bringing together two of the most desirable features in pharmaceutical market. On one hand, it is especially preferred by people with a high propensity for skin irritation, such as patients undergoing chemotherapy [15]. On the other hand, $\mathrm{TiO}_{2}$ is necessary for manufacturing sunscreens with a high SPF [15]. Its properties ensure that formulations can be uniformly spread on the skin, granting a better UV protection. Therefore, $\mathrm{TiO}_{2}$ can combine both stability and SPF of the sunscreen formulation. However, following the recommendations of the European Commission Recommendation of 22 September 2006, the protection factor against UVA should be at least one-third of the overall SPF. Thus, taking into account that $\mathrm{TiO}_{2}$ is primarily a UVB absorbing compound, it is important to add another physical filter with a complementary protection. As $\mathrm{ZnO}$ is more effective in the UVA range, the combination of both filters will assure a broad-band UV protection [16].

Regarding the use of chemical filters in sunscreen formulation, it is becoming less popular nowadays due to the possible harmful effects. Thus, in order to find effective topical photoprotective agents, plantderived products including natural oils have been gaining significant attention due to their safety and multiple biological activities on the skin and cost effectiveness. In this context, green coffee oil, a rich source of antioxidants and polyphenols, has arisen as a potential candidate to replace the chemical filters in "green" sunscreen formulations [17].

Therefore, the major aim of this research study was to develop and fully characterize an innovative sunscreen formulation based on the Pickering emulsion concept, i.e., surfactant-free emulsions stabilized by SP and physical UV filters associated to natural oils as a key strategy for achieving the UV-induced skin damage prevention.

\section{Material and Methods}

\subsection{Materials}

Carrot oil, Brazil nut oil and refined raspberry seed oil and avocado oil were kind gifts from Provital Group (Barcelona, Spain), Croda do
Brasil Ltda. (Campinas, Brazil), Seatons (East Yorkshire, UK) and BioChemica (Melbourne, USA), respectively. Green Coffee Oil (GCO) was supplied by COOXUPÉ - Cooperativa de Cafeicultores de Gauxupé (Minas Gerais, Brazil). Spent coffee oil (SCO) were supplied by NovaDelta - Comércio e Indústria de Cafés, S.A. (Campo Maior, Portugal). Triethoxycaprylylsilane rutile titanium dioxide (Unipure White LC 987) was a gift from Sensient (Milwaukee, USA). Aluminum starch octenylsuccinate (DryFlo® Plus) was obtained from AkzoNobel (Amsterdam, Netherlands). Zinc Oxide (Tego ${ }^{\circledR}$ Sun. Z 500) was obtained from Evonik Industries AG (Essen, Germany). Liquid paraffin was obtained from José Vaz Pereira, S.A. (Lisbon, Portugal). Ethanol was obtained from Merck ${ }^{\circledR}$ (Kenilworth, USA). All other reagents were HPLC grade. Purified water was obtained by reverse osmosis (Millipore, Elix 3).

\subsection{Methods}

\subsubsection{Solid Particles -TiO2, $\mathrm{ZnO}$ and Aluminum Starch Octenylsuccinate (ASt)}

2.2.1.1. Wettability Measurement. Contact angles of water and green coffee oil on $\mathrm{ZnO}$, TiO2 and ASt in air atmosphere were measured at room temperature by using ConAnXL - a Microsoft Excel based workbook and add-in software (freely available upon request) as described in detail elsewhere [18]. All measurements were performed in triplicate.

2.2.1.2. Particle Size of Solid Particles. Particle size distribution was determined using a Malvern Mastersizer 2000 (Malvern Instruments, UK), coupled with a Hydro S accessory. The refractive index was 1.52 (default). Data was expressed in terms of relative distribution of volume of particles in the range of size classes, and given as diameter values corresponding to percentiles of 10,50 and 90 . The Span value is a useful statistical parameter to characterize the particle size distribution.

\subsubsection{Natural Oils}

2.2.2.1. In Vitro SPF Determination. All natural oils were accurately weighed $(0.25 \mathrm{~g})$, diluted with ethanol absolute, followed by ultrasonication for $5 \mathrm{~min}$ and filtered through filter paper (Whatman тм 42). The absorption spectra of samples solution were obtained in the range of 290 to $320 \mathrm{~nm}$ (Hitachi U-2001, USA) every $5 \mathrm{~nm}$, using a standard $1 \mathrm{~cm}$ quartz cell, and ethanol as the blank reagent. Triplicates were made, followed by the application of the Mansur equation Eq. (1).

$\mathrm{SPF}_{\text {spectrophotometric }}=\mathrm{CF} \times \sum_{290}^{320} \mathrm{EE}(\lambda) \times \mathrm{I}(\lambda) \times \operatorname{Abs}(\lambda)$

where $E E(\lambda)$ is the erythemal effect spectrum; $I(\lambda)$ is the solar intensity spectrum; Abs $(\lambda)$ is absorbance of sunscreen product; CF is the correction factor $(=10)$ [9]. The values of $\mathrm{EE} \times \mathrm{I}$ are constants determined by Sayre [19].

\subsubsection{Sunscreen Formulations - PhotoStarch 1 (PS1) and PhotoStarch 2} (PS2)

2.2.3.1. Preparation of the PS1 and PS2. According to the pre-formulation studies (results not shown), two final formulations were selected (Table 1 ) based on macroscopic appearance, stability and SPF value. The continuous oil phase (Phase A) consisted of green coffee oil, and the aqueous phase (Phase $\mathrm{B}$ ) was composed by purified water and ethanol. Solid particles - $\mathrm{TiO}_{2}, \mathrm{ZnO}$ and ASt were firstly dispersed in the oil phase. The oil and aqueous phases were then mixed using a high-speed homogenizer (UltraTurrax®, IKA-Werke GmbH \& Co. KG, Germany) at room temperature (cold process). 
Table 1

Qualitative and quantitative composition of the final formulations.

\begin{tabular}{llr}
\hline Ingredients & \multicolumn{2}{l}{$\begin{array}{l}\text { Quantitative } \\
\text { composition (\%, w/w) }\end{array}$} \\
\cline { 2 - 2 } & \multicolumn{1}{l}{ PS1 } & PS2 \\
\hline Phase A (external) & & \\
Triethoxycaprylylsilane titanium dioxide & 20 & 20 \\
Zinc oxide & 15 & 15 \\
Aluminum starch octenylsuccinate & - & 5 \\
Green coffee oil & 35 & 35 \\
Phase B (internal) & & 6 \\
Ethanol & 6 & 19 \\
Purified water & 24 & \\
\hline
\end{tabular}

\subsubsection{Efficacy of the Sunscreen Formulations}

2.2.4.1. In Vitro SPF Determination. The SPF was assessed using the Optometrics SPF-290S Analyzer (Optometrics Corporation, Essex, UK). The samples were prepared by spreading $110 \mathrm{mg}$ of each formulation over a Transpore ${ }^{\circledR}$ tape $(70.7 \times 70.7 \mathrm{~mm})$ to obtain a film of $2 \mathrm{mg} / \mathrm{cm}^{2}$, as specified by the European legislation [20]. Each sample was exposed to UVB and UVA radiation provided by a 125 W CW xenon arc lamp. The xenon arc lamp assured the constant spectrum and the high UV power output.

The analyzer performed scans in 12 different spots on the Transpore ${ }^{\circledR}$ tape substrate. Each scan takes a transmittance $(\mathrm{T})$ measurement every $2 \mathrm{~nm}$ from a wavelength ranging from 290 to $400 \mathrm{~nm}$. The Monochromatic Protection Factor (MPF) was determined for the selected wavelengths using Eq. (2). The SPF value was calculated using Eq. (3).

$\mathrm{MPF}=\frac{1}{\mathrm{~T}}$

$\mathrm{SPF}=\frac{\sum_{290}^{400} \mathrm{E} \lambda \cdot \mathrm{B} \lambda}{\sum_{290}^{400} \frac{\mathrm{E} \lambda \cdot \mathrm{B} \lambda}{\mathrm{MPF} \lambda}}$

where, (E) is the spectral irradiance of terrestrial sunlight under controlled conditions and (B) is the erythema effectiveness [21].

2.2.4.2. In Vitro Sunscreen Water Resistance. The water resistance of developed sunscreens was measured using an improved in vitro bath system. An amount of $2 \mathrm{mg} / \mathrm{cm}^{2}$ of sunscreen formulation was dispensed onto the plate, and carefully applied with a rubber-gloved finger. After drying for $15 \mathrm{~min}$, the SPF of each sample was determined using the SPF 290 analyzer (Optometrics SPF-290S Analyzer). The samples were immersed in the in vitro bath system $\left(29 \pm 2{ }^{\circ} \mathrm{C}\right)$ and washed away by the water flow (150 rpm) during $20 \mathrm{~min}$. The samples were allowed to air dry for $15 \mathrm{~min}$ and SPF was measured again. The samples were immersed once more and washed during $20 \mathrm{~min}$. The samples were allowed to air dry for $15 \mathrm{~min}$ and SPF was measured to calculate the water resistance retention (\%WRR) of the sunscreens, as defined by Eq. (4).

$\% \mathrm{WRR}=\frac{\mathrm{SPF}_{\mathrm{wet}}}{\mathrm{SPF}_{\mathrm{dry}}} \times 100$

where, $\mathrm{SPF}_{\text {dry }}$ and $\mathrm{SPF}_{\text {wet }}$ are the SPFs before and after water immersion, respectively $[22,23]$.

2.2.5. Physical and Microbiological Stability of Sunscreen Formulations

The PS1 and PS2 emulsions were stored during 3 months at room temperature $\left(25 \pm 2{ }^{\circ} \mathrm{C}\right)$ and under accelerated conditions $\left(40 \pm 2^{\circ}\right.$ C). Samples were analysed for physical (macroscopic appearance, $\mathrm{pH}$ by potentiometry and droplet size distribution) and microbiological stability before the storage period and after 14 days, 1 and 3 months.

2.2.5.1. Droplet Size Distribution. After 1 day at room temperature, the emulsions were observed using an optical microscope (Olympus CX40, Japan) equipped with a video camera. One drop of each emulsion was added to a glass slide without covering glass, and diluted with two drops of green coffee oil. The droplet size was determined using the image analysis software Olympus Stream Essentials ${ }^{\circledR}$. The size data was expressed in terms of relative size distribution of particles, and given as diameter values corresponding to percentiles of 50\% [24].

2.2.5.2. Microbiological Stability. The microbiological stability was performed according to the ISO 16212:2008, ISO 21149:2006 and ISO 21148:2005 [25-27].

\subsubsection{Characterization Studies of Sunscreen Formulations}

\subsubsection{Structural Analysis}

2.2.6.1.1. Dynamic Viscosity. Shear rate vs. shear stress measurements were performed using a AR 2000ex rheometer (TA Instruments, USA). Rotational viscosity was determined using a cone geometry with an angle of $2^{\circ}$. Flow curves were generated by ramping the shear rate from 0 to $100 \mathrm{~s}^{-1}$ (ascent curve) and then from 100 to $0 \mathrm{~s}^{-1}$ (descent curve) for $120 \mathrm{~s}$ each curve. All tests were performed on $1 \mathrm{~g}$ samples at $25.0 \pm 0.5^{\circ} \mathrm{C}$, in duplicate.

2.2.6.1.2. Oscillatory Measurements. Oscillatory measurements were performed to investigate the behaviour of these formulations when subjected to small deformations. Oscillation frequency sweep tests were performed from 0.1 to $100 \mathrm{~Hz}$. Viscoelastic experiments were carried out by exposing the samples to a forced oscillation deformation. Prior to the oscillation tests, the sweep tests were conducted at $1 \mathrm{~Hz}$ and 0 to $50 \mathrm{~Pa}$ (stress sweep test), and at 0.1 to $100 \mathrm{~Hz}$ and $1 \mathrm{~Pa}$ (frequency sweep test) for both emulsions. The creep and recovery tests were carried out with 1 Pa shear stress, allowing 360 s for creep and other $360 \mathrm{~s}$ for relaxation. All tests were performed on $1 \mathrm{~g}$ samples at $25.0 \pm 0.5^{\circ} \mathrm{C}$ in duplicate.

2.2.6.2. Texture Profile Analysis (TPA). A Texture Analyzer TA.XT Plus (Stable Micro Systems Ltd., Surrey, UK) was used to examine textural characteristics of the emulsions (hardness, elasticity, compressibility, adhesiveness and cohesiveness). The TPA mode was carried out using an analytical probe (P/10, $10 \mathrm{~mm}$ Delrin), which was twice depressed into the sample at a defined rate $(5 \mathrm{~mm} / \mathrm{s})$ to a desired depth (15 mm), allowing $15 \mathrm{~s}$ of delay between consecutive compressions. The samples were placed into cylindrical tubes with the same dimensions (at a fixed height). Six replicates were performed at $25{ }^{\circ} \mathrm{C}$ for each formulation. Data collection and calculation were performed using the Texture Exponent 3.0.5.0 software package of the instrument.

\subsubsection{In Vitro EpiSkin®}

The validated reconstructed human epidermis EpiSkin ${ }^{\circledR}$ skin irritation test method was used [28]. The EpiSkin ${ }^{\circledR}$ tissues were supplied by SkinEthic Laboratories (www.skinethic.com) consisting in a reconstructed organotypic culture of adult human keratinocytes reproducing a multilayered and well differentiated epidermis.

The method used following the instruction of the producer, the 12 well plates, containing 12 inserts of tissues $\left(0.38 \mathrm{~cm}^{2}\right)$, were transferred into 12 wells plates containing $2 \mathrm{~mL}$ of maintenance medium and incubated at $37{ }^{\circ} \mathrm{C}$ ( $5 \% \mathrm{CO} 2,>95 \%$ humidity). After $24 \mathrm{~h}$, the second column of each plate was filled with maintenance medium preheated at $37^{\circ} \mathrm{C}$.

An amount of $10 \mathrm{mg}$ of both formulations were applied directly and contacted during $15 \mathrm{~min}$ with the epidermis samples. Phosphate buffer saline (PBS) was used as negative control and sodium dodecyl sulfate (SDS) (5\% in distilled water) as positive control. 
Cell viability was determined with the MTT assay. Tissues were transferred to wells containing $2 \mathrm{~mL}$ of a $0.3 \mathrm{mg} / \mathrm{mL}$ MTT solution and incubated for $3 \mathrm{~h}\left(37^{\circ} \mathrm{C}, 5 \% \mathrm{CO}_{2}, 95 \%\right.$ humidified atmosphere). After incubation, the epidermis tissues were put in contact with acidic isopropanol ( $0.5 \mathrm{~mL} /$ tube) to extract the intracellular formazan.

The tubes were incubated for $4 \mathrm{~h}$ in dark with periodic vortexing, after that, a duplicate of $200 \mu \mathrm{L}$ was transferred to a 96-well flat bottom microtiter plate. Absorbance was read at $570 \mathrm{~nm}$ with acidified isopropanol as blank and viability was calculated considering $100 \%$ for the negative control.

\subsubsection{In Vivo Studies of Sunscreen Formulations}

2.2.8.1. Human Repeat Insult Patch Test (HRIPT). A safety evaluation study was performed on sunscreen formulations, using the Marzully and Maibach [29] HRIPT protocol. Briefly, the product was applied on the back of 50 healthy volunteers that previously signed the informed written consent. Subjects with dermatological or other medical or physical conditions precluding the topical application of the testing product were excluded, along with pregnant and nursing women. For the induction period of a possible allergic reaction, a series of 9 patches (Finn Chamber standard) were performed over a period of 3 weeks. An occlusive patch containing $20 \mathrm{mg}$ of the formulation was applied on the left side of the back where it remained for $48 \mathrm{~h}$. After that period, the patch was removed, the skin was evaluated and a new patch was applied. Reactions after patching were scored according to the recommendations of the International Contact Dermatitis Research Group [30].

A 2 weeks rest period was followed without application of the testing product. During the challenge period, new patches were prepared and fixed in the same manner as in the induction period, but also on the right side of the back (i.e. a virgin site). The patches were removed after $48 \mathrm{~h}$ and the skin reactions were evaluated as before at 48,72 , and $96 \mathrm{~h}$ after patching using the same scoring system.

This protocol was approved by the local Ethical Committee and respected the Helsinki Declaration and the AFSSAPS regulations on performed HRIPT studies on cosmetic products. The study was conducted under the supervision of a dermatologist who participated in the evaluation of irritation/allergic reactions to the tested formulations.

2.2.8.2. In Vivo Sunscreen Water Resistance. The water resistance of sunscreens was tested on 3 subjects (Fitzpatrick skin type II). In the testes, panellists would cleanse their forearms using a mild cleanser and leave them to air dry for $30 \mathrm{~min}$ before starting the test. Initial cross polarized images are taken after the sunscreens application $\left(2 \mathrm{mg} / \mathrm{cm}^{2}\right)$ on the inner forearm $\left(4 \mathrm{~cm}^{2}\right)$.

The amount of each sunscreen formulation left before and after water bath immersion was quantified via cross-polarized imaging by means of the Visia ${ }^{\circledR}$ CA (Canfield Scientific, Faitfield, NJ). Panellists immerse their forearms into a water bath system $\left(29 \pm 2{ }^{\circ} \mathrm{C}\right)$ and washed away by the flow of water ( $150 \mathrm{rpm}$ ) during $40 \mathrm{~min}$ [23]. Their forearms were allowed to air dry for 15 min and the amount of sunscreen was measured again. This procedure was repeated and the amount of sunscreen was measured again to calculate the water resistance of the sunscreen formulations.

This method yielded a series of three cross-polarized images for each panellist: clean skin (without sunscreen), immediately after sunscreen application, and post water bath. Water resistance information was obtained from the cross-polarized Visia ${ }^{\circledR}$ CA imaging mode in visible light. The RGB colour space of the raw bitmap images was converted to relative luminance using Image ${ }^{\circledR}$. From these images, average $\mathrm{L}$ changes for each sunscreen area were obtained from histograms. Skin whiteness was defined as the change in L value before and after water immersion, and the percentage of water resistance retention (\%WRR) of the sunscreens was determined according to Eq. (5).

$\% \mathrm{WRR}=\frac{\mathrm{L}_{\text {washed protector }}-\mathrm{L}_{\text {skin }}}{\mathrm{L}_{\text {protector }}-\mathrm{L}_{\text {skin }}}$

\subsubsection{Statistical Analysis}

The data were expressed as mean and standard deviation (mean \pm SD) of experiments. Statistical evaluation of data was performed using one-way analysis of variance (ANOVA). Tukey-Kramer multiple comparison test (GraphPad PRISM 5 software, USA) was used to compare the significance of the difference between the groups $(p<0.05)$.

\section{Results and Discussion}

\subsection{Solid Particles}

The solid particles chosen to formulate the PhotoStarch (PS) Pickering emulsions were $\mathrm{TiO}_{2}, \mathrm{ZnO}$ and ASt particles. $\mathrm{TiO}_{2}$ is a common UVB filter used for manufacturing sunscreens with a high SPF [15]. Previous results clearly demonstrated the importance of the presence of $\mathrm{TiO}_{2}$ attending that this solid particle (mainly at 20\% concentration) contributed for emulsion stability and high SPF (data not shown). The addition of another filter ( $\mathrm{ZnO}$ ) was intended to ensure an adequate protection in the UVA range. Therefore, the combination of these filters resulted in a broad-band UV protection [16], while improving the antibacterial and antifungal properties of the formulations [31]. ASt is a modified starch used in pharmaceutical and personal care products at concentrations up to $30 \%$ as an anticaking and a viscosity increasing agent [13], which can also function as a steric stabilizer. However, in the present work ASt was not used for its stabilizer properties but rather as SPF enhancer of PS Pickering emulsions (Table 4).

\subsubsection{Wettability Measurements}

In Pickering emulsions, one of the liquid phases will probably wet the solid more than the other liquid, being the latter the disperse phase. The importance of the wettability of the particles at the oilwater interface is quantified by the contact angle $(\theta)$, which will determine the emulsion type. If the contact angle measured through the aqueous phase is $<90^{\circ}$, the emulsion will be o/w and, by contrast, if the contact angle is $>90^{\circ}$, the emulsion will be w/o. Furthermore, w/o emulsions are more water resistant and provide higher SPF at the same UV filters concentration than o/w emulsions [32].

According to Table $2, \mathrm{TiO}_{2}, \mathrm{ZnO}$ and starch particles stabilize more effectively w/o emulsions. All solid particles had a contact angle with water $>90^{\circ}$, and simultaneously, a contact angle with paraffin and green coffee oil $<90^{\circ}$ thus, allowing the formulation of a stable emulsion by combining these three types of particles.

Table 2

Contact angle of water, liquid paraffin and green coffee oil with TiO2, ZnO and ASt and particle size distribution of the different solid particles proposed (mean \pm SD, $n=6$ ).

\begin{tabular}{|c|c|c|c|c|c|c|c|}
\hline \multirow[t]{2}{*}{ Samples } & \multicolumn{3}{|c|}{ Contact angle $\left({ }^{\circ}\right)$} & \multicolumn{4}{|c|}{ Particle size distribution $(\mu \mathrm{m})$} \\
\hline & Water & Liquid paraffin & Green coffee oil & Span & $\mathrm{d}(0.1)$ & $\mathrm{d}(0.5)$ & $\mathrm{d}(0.9)$ \\
\hline $\mathrm{TiO}_{2}$ & $106.5 \pm 0.7$ & 0 & $60.1 \pm 1.9$ & $37.4 \pm 1.4$ & $0.1 \pm 0.1$ & $0.2 \pm 0.1$ & $7.1 \pm 0.3$ \\
\hline $\mathrm{ZnO}$ & $100.2 \pm 2.6$ & 0 & $23.3 \pm 0.1$ & $19.5 \pm 5.5$ & $0.2 \pm 0.1$ & $0.6 \pm 0.3$ & $11.4 \pm 3.7$ \\
\hline ASt & $109.0 \pm 0.4$ & $15.8 \pm 1.1$ & 0 & $1.0 \pm 0.1$ & $7.3 \pm 0.1$ & $13.52 \pm 0.1$ & $20.8 \pm 0.0$ \\
\hline
\end{tabular}


Table 3

$\mathrm{SPF}$ found for the natural oils (mean $\pm \mathrm{SD}, n=3$ ).

\begin{tabular}{ll}
\hline Sample & SPF \\
\hline Raspberry oil & $0.48 \pm 0.18$ \\
Avocado oil & 0 \\
Carrot oil & $0.64 \pm 0.06$ \\
Green coffee oil & $5.03 \pm 0.23$ \\
Spent coffee oil & $1.57 \pm 0.07$ \\
Brazil nut oil & $0.02 \pm 0.05$ \\
\hline
\end{tabular}

\subsubsection{Particle Size Distribution}

It is important to prove that the particles have a size $>100 \mathrm{~nm}$ in order to ensure an environmentally friendly cosmetic product. According to the international "green" standards, nanomaterials are forbidden. Particle size distributions of $\mathrm{TiO} 2, \mathrm{ZnO}$ and ASt showed that all particles were larger than $100 \mathrm{~nm}$, as requested to avoid regulatory issues (Table 2).

\subsection{Natural Oils}

\subsubsection{SPF Measurement}

Sunscreens are usually composed of synthetic chemical filters with a high capacity to absorb sun light at the region of UVB (320-290 nm) and UVA (400-320 nm) spectrum. The reduction of filter concentration in sunscreen formulations is a strategy to improve quality without affecting their properties as well as to reduce the adverse effects (e.g. estrogenic effects, disruption of human endocrine activity, etc.).

Lately, natural ingredients have been attracting significant attention, and they are chemical compound or substance produced by a living organism founded in nature. The increasing association of natural ingredients with antioxidant activity may also improve the photoprotective activity of sunscreen formulations. In fact, these natural ingredients present several advantages, such as the bioactivity, relative safety, and obtainment from renewable sources, low cost, besides the feasibility for application in a wide range of health care products, and are obtained from renewable sources [33]. Natural ingredients are a viable source of bioactive compounds, such as polysaccharides and polyphenols, namely, phenolic acids (chlorogenic acid, caffeic acid and p-coumaric acid, among others) and alkaloids, which can be applied in the pharmaceutical, food and cosmetic industries [34].

Several natural ingredients, with more or less complex chemical structures, have been found to possess antioxidant activity. Determination of antioxidant compounds from cosmetics is often difficult due to the matrix complexity. In fact, natural ingredients usually occur as a combination of numerous types of bioactive compounds with different properties. However, their separation still remains a big challenge for the process of identification and characterization of bioactive compounds. Thus, it is necessary to develop performant methods of analysis, which can exactly, and with precision determine the type and amount of bioactive compounds present in cosmetic products [17,35].

In this context, GCO, a rich source of antioxidants and polyphenols, has arisen as a potential candidate to replace the chemical filters in sunscreen PS formulations [17]. The SPF determination for different natural oils is represented on Table 3.
The pure GCO showed the highest SPF $(\simeq 5)$, which is in line with previous results suggesting the use of GCO as a potential natural product for improving SPF in sunscreens formulations thus allowing to decrease the concentration of chemical filters in such formulations [36]. For example, GCO showed a high synergistic effect when associated with a synthetic sunscreen as ethylhexylmethoxycinnamate, leading to an increase of $20 \%$ in SPF [36]. Therefore, this promising oil was used as the external phase of the final PS formulations.

\subsection{Sunscreen Formulations - PS1 and PS2}

Attending to pre-formulation studies (results not shown), two formulations (PS1 and PS2) were fully characterized and selected for further assessment. Although the only variable between these two emulsions was the presence (PS2) or absence (PS1) of starch, the respective results were surprisingly different.

\subsubsection{SPF Measurement}

Based on the results showed on Table 4, both emulsions showed high values of SPF with a suitable UVA/UVB ratio. Although, starch had shown no photoprotection property by itself, there was a synergistic increase of SPF value (around 2 fold) when combined with physical filters as $\mathrm{TiO}_{2}$ in PS2 formulation. Other authors also reported that the addition of $5 \%$ starch induced a SPF enhancement of $40 \%$ [37]. Thus, it was possible to reduce the amount of physical filters by including starch in the sunscreen formulation [38].

Differences in SPF between PS1 and PS2 formulations were only detected in the in vitro determinations (Optometrics SPF-290S Analyzer). However, it must be noticed that this evaluation has some limitations. According to Pissavini et al. [39] high SPF values are more difficult to measure due to the biological variability. Another limitation is the impossibility to detect biological effects that might affect the SPF determination, since it is a simple physical measurement. For example, a substance exhibiting antioxidant and anti-inflammatory activities may delay the appearance of erythema, which cannot be observed under in vitro conditions.

Therefore, the Real-Life SPF is usually lower than the SPF obtained with the solar simulator as here observed. These SPF values would only be the same in the case of the ideal sunscreen with spectral homeostasis.

\subsubsection{In vitro Sun. Product Water Resistance}

The protection that sunscreens provide against sunburn is neither absolute nor tight. The photoprotection conferred by a sunscreen is extremely variable in a context of beach or pool since there are many conditions that contribute to its decline (e.g. the trips to the bathroom or the simple contact with the towel will decrease the sunscreen coverage). That is why it is strongly recommended to reapply the sunscreen after prolonged swimming or vigorous activity. One of the many factors that can have an effect on the level of protection is the water contact because UV absorbers present in the formulation can leach out or be physically removed by the washing action [22].

In vivo water resistance testing of topical sunscreens is time consuming, expensive and experimentally problematic [40]. Thus, initially it was opted to determine the sun product water resistance under in vitro conditions. This method measures the SPF following a defined

Table 4

In vitro and in vivo efficacy tests of the PhotoStarch emulsions.

\begin{tabular}{|c|c|c|c|c|c|c|}
\hline \multirow[t]{2}{*}{ Formulations } & \multicolumn{3}{|c|}{$\begin{array}{l}\text { In vitro sun protection factor (SPF) - optometrics } \\
\text { SPF-290S analyzer }\end{array}$} & \multicolumn{2}{|c|}{ In vitro sun product water resistance } & \multirow{2}{*}{$\begin{array}{l}\text { In vivo sun product } \\
\text { water resistance } \\
\text { \%WRR* }\end{array}$} \\
\hline & SPF & UVA/UVB & UVA & $\% \mathrm{WRR}^{*}$ after $1 \mathrm{st}$ immersion & $\%$ WRR* after 2 nd immersion & \\
\hline PS1 & $43.4 \pm 6.2$ & $0.9 \pm 0.1$ & $33.0 \pm 5.6$ & $55.3 \pm 6.9$ & $54.7 \pm 7.6$ & $70.0 \pm 2.0$ \\
\hline PS2 & $82.3 \pm 10.3$ & $0.9 \pm 0.1$ & $71.1 \pm 10.1$ & $62.6 \pm 4.3$ & $50.7 \pm 6.1$ & $71.2 \pm 0.5$ \\
\hline
\end{tabular}

\footnotetext{
* WRR - water resistance retention.
} 
water immersion procedure. A product is considered water resistant when the value for the lower $90 \%$ one-sided confidence limit has to be greater than or equal to 50\% [22]. Analysis of Table 4 revealed that PS1 and PS2 formulations showed an in vitro \%WRR $\geq 50 \%$ after both immersions. Consequently, it was possible to ensure the water resistance claim of both formulations.

\subsubsection{Physical and Microbiological Stability of Sunscreen Formulations}

The stability of these emulsions in terms of $\mathrm{pH}$ and macroscopic characteristics was assessed for 3 months at room temperature $\left(25 \pm 2{ }^{\circ} \mathrm{C}\right)$ and under accelerated conditions $\left(40 \pm 2{ }^{\circ} \mathrm{C}\right)$. After this period, both PS1 and PS2 emulsions remained white with a creamy and homogeneous aspect for both storage conditions. In fact, no instability-related processes, such as creaming (or sedimentation), flocculation, coalescence or phase inversion were observed. The $\mathrm{pH}$ values $(\mathrm{pH} \sim 5$ ) did not significantly vary over time as well as the droplet size (Table 5).

Microbiological testing revealed these formulations were stable for 3 months as the total aerobic microbial, yeast and mould count presented accepted values following the established criteria $(<10 \mathrm{cfu} / \mathrm{g})$.

\subsubsection{Characterization Studies of Sunscreen Formulations}

3.3.4.1. Structural Analysis. The physical characterization of PS1 and PS2 emulsions has been performed using several techniques crucial for the predictive performance of the product under a variety of conditions, particularly during product filling, spreadability on the skin and easiness of product removal from the final packaging system. The flow curves (Fig. 1 (a)) showed that the PS emulsions were non-Newtonian fluids, which means their viscosity is dependent on the shear rate.

These emulsions were also characterized as shear thinning fluids, since their structure need an initial tension to be deformed and start flowing (yield stress), and after that, a non-linear curves obtained as the apparent viscosity decreased with increased stress [41]. In addition, the relation between the shear stress and the shear rate was also timedependent, particularly in the case of PS2.

Considering the time during which these formulations were submitted to different forces, it was possible to verify that the apparent viscosity was not only dependent of the shear rate magnitude, but also of the time of this shear rate application. In this context, the PS1 and PS2 emulsions exhibited rheopectic behaviours. However, at lower shear rates (around $30 \mathrm{~s}^{-1}$ ), this behaviour was altered and both emulsions began to exhibit thixotropic behaviour [41].

The shear stress sweep precedes the frequency sweep and creep/ recovery tests, making it possible to determine the values of shear stress within a linear range at which the sample does not suffer any deformation. Both emulsions were not disrupted at 0 to $5 \mathrm{~Pa}$. Thus, the value of $\mathrm{G}^{\prime}$ (elastic modulus) and $\mathrm{G}^{\prime \prime}$ (viscous modulus) remained linear within this region of linear viscoelasticity, indicating the suitable shear stress to be used in frequency sweep and creep/ recovery tests.

Both emulsions exhibited a higher $G^{\prime}$ than $G^{\prime \prime}$ values ( 1 (b)), a gel like behaviour [42]. The frequency sweep curves of PS emulsions showed that there was practically no variation of the elastic and viscous moduli at the tested range $(0.1-100 \mathrm{~Hz})$.

Table 5

Droplet size distribution of the P1 and PS2 emulsions ( $n=625$; mean \pm SD) in batches 1 and 2 (mean $\pm \mathrm{SD} ; n=3$ ) stored at $25^{\circ} \mathrm{C}$ and $40{ }^{\circ} \mathrm{C}$ during 90 days.

\begin{tabular}{cclll}
\hline & Time (days) & Temperature $\left({ }^{\circ} \mathrm{C}\right)$ & PS1 & PS2 \\
\hline Droplet size distribution & 1 & 25 & $6.2 \pm 4.1$ & $5.7 \pm 4.3$ \\
$\mathrm{~d}(0.5)(\mu \mathrm{m})$ & 14 & 25 & $6.4 \pm 5.8$ & $5.6 \pm 6.3$ \\
& & 40 & $7.1 \pm 8.5$ & $5.3 \pm 3.8$ \\
& \multirow{2}{*}{30} & 25 & $5.9 \pm 7.2$ & $6.5 \pm 5.7$ \\
& \multirow{2}{*}{90} & 40 & $6.7 \pm 3.4$ & $6.2 \pm 5.9$ \\
& 25 & $6.4 \pm 3.9$ & $6.1 \pm 6.2$ \\
& 40 & $5.7 \pm 4.1$ & $5.8 \pm 3.9$
\end{tabular}

When a viscoelastic material has a storage (or elastic) modulus higher than the viscous (or loss) modulus, the shear energy is temporarily stored during the test and can be retrieved later, as usually occurs in emulsion systems. Emulsion systems with this feature usually exhibit a high stability [43].

When the emulsions were submitted to predetermined shear stress (1 Pa for both emulsions) for $150 \mathrm{~s}$ (creep), and then left for another $150 \mathrm{~s}$ without shear stress (recovery), both P1 and PS2 emulsions suffered deformation according to the compliance value (J) (Fig. 1 (c)). In the recovery part of this study, the samples could recover part of their former structure, and the elastic part of the deformation was reversed.

Regarding the TPA results, PS1 and PS2 emulsions demonstrated a wide range of mechanical properties dependent on the presence of starch (Table 6). In fact, the starch affected the mechanical behaviour of these formulations, which may influence their adhesion on the skin.

(a)

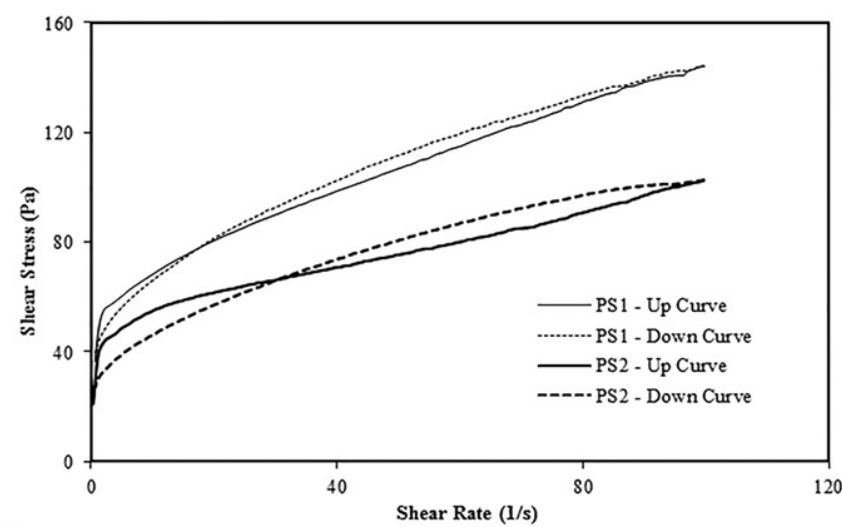

(b)

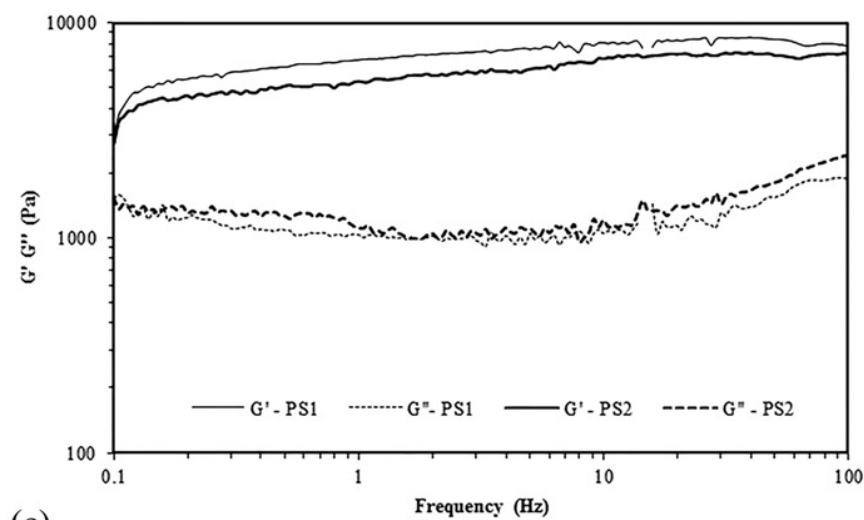

(c)

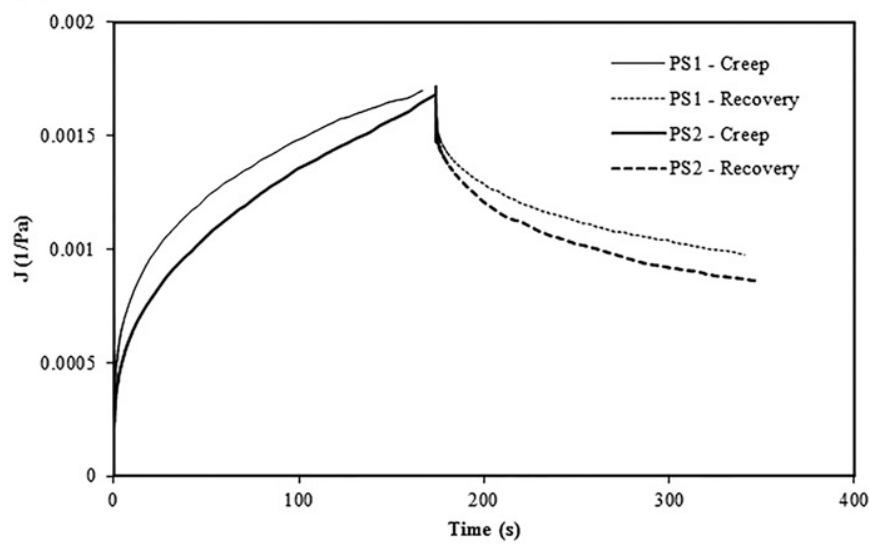

Fig. 1. Flow curves (a), frequency sweep plot (b) and creep and recovery plot (c) of PS1 and PS2 emulsions. 
Table 6

Mechanical properties of the emulsions extracted from the TPA mode (mean $\pm \mathrm{SD}, n=3$ ).

\begin{tabular}{|c|c|c|c|c|c|c|}
\hline \multicolumn{2}{|c|}{ Formulations } & \multirow{2}{*}{$\frac{\text { Hardness }(\mathrm{g})}{16.7 \pm 0.1}$} & \multirow{2}{*}{$\begin{array}{l}\text { Adhesiveness }(|\mathrm{g} \cdot \mathrm{s}|) \\
26.9 \pm 0.1\end{array}$} & \multirow{2}{*}{$\begin{array}{l}\text { Elasticity } \\
1.0 \pm 0.1\end{array}$} & \multirow{2}{*}{$\frac{\text { Cohesiveness }}{0.8 \pm 0.1}$} & \multirow{2}{*}{$\begin{array}{l}\text { Compressibility }(\mathrm{g} \cdot \mathrm{s}) \\
21.4 \pm 0.6\end{array}$} \\
\hline PS1 & Batch 1 & & & & & \\
\hline & Batch 2 & $16.0 \pm 0.8$ & $27.3 \pm 0.2$ & $1.0 \pm 0.1$ & $0.8 \pm 0.1$ & $22.0 \pm 1.9$ \\
\hline \multirow[t]{2}{*}{ PS2 } & Batch 1 & $11.2 \pm 0.2$ & $13.0 \pm 0.6$ & $1.0 \pm 0.1$ & $0.8 \pm 0.1$ & $22.0 \pm 0.1$ \\
\hline & Batch 2 & $11.8 \pm 0.7$ & $13.9 \pm 0.6$ & $1.0 \pm 0.1$ & $0.8 \pm 0.1$ & $17.6 \pm 0.2$ \\
\hline
\end{tabular}

The addition of starch caused a decrease in emulsion hardness. In contrast, PS1 emulsion (without starch) showed a greater hardness. In other words, the starch addition influenced positively this parameter. The same trend was verified for adhesiveness, which is more related to surface characteristics, and depends on a combined effect of adhesive and cohesive forces. PS2 emulsion showed a lower value of adhesiveness, which was again dependent on the presence of starch, since this was the only different ingredient between the two formulations.

Considering the elasticity, cohesiveness and compressibility results, the presence or absence of starch did not influence these parameters. So, the only parameters altered by the presence of starch were hardness and adhesiveness, attending to the decrease in both values.

In summary, the addition of ASt promoted a reduction in the apparent viscosity of the PS2 emulsion. In fact, the presence of starch can improve the spreadability of the emulsions over the skin surface. Considering the sunscreen formulation, a maximized photoprotection will be obtained by covering the total skin area. These phenomena can be explained by the triethoxycaprylylsilane and aluminum octenylsuccinate surface treatment of $\mathrm{TiO}_{2}$ and ASt, respectively (Fig. 2). According to other authors, the silane coating decreases the interaction between the alumina aggregates, present on the surface of the starch granules, decreasing the shear stress required to break the flocculation [44]. Thus, both $\mathrm{TiO}_{2}$ and ASt, surface treatment can interact results in a lower viscosity.

\subsubsection{In Vitro EpiSkin ${ }^{\circledR}$}

The in vitro safety topical use of PS emulsions was tested on reconstituted human epidermis by the EpiSkin ${ }^{\circledR}$ model and is useful to classify skin irritants able to produce a reduction in cell viability [45]. The tissue viability measured as optical density by the MTT assay and calculated as percentage of cytotoxicity compared to the negative control (PBS), was $84.0 \pm 5.0 \%$ and $81.0 \pm 4.0 \%$ for PS1 and PS2, respectively, whereas for the positive control (SDS) it was $36.0 \pm 4.0 \%$. A product is considered an irritant when viability is reduced by $50 \%$.

The absence of skin-irritant effects at the concentrations tested indicated that both formulations could be safe for topical use.

\subsubsection{In Vivo Studies of Sunscreen Formulations}

3.3.6.1. Human Repeat Insult Patch Test (HRIPT). The HRIPT assay intends to assure two essential conditions: the first one is the skin compatibility and the second one, the absence of allergenic potential of the tested formulations. Therefore, HRIPT was conducted to justify the claim "dermatological tested".

\section{Emulsion stabilised by solid particles}

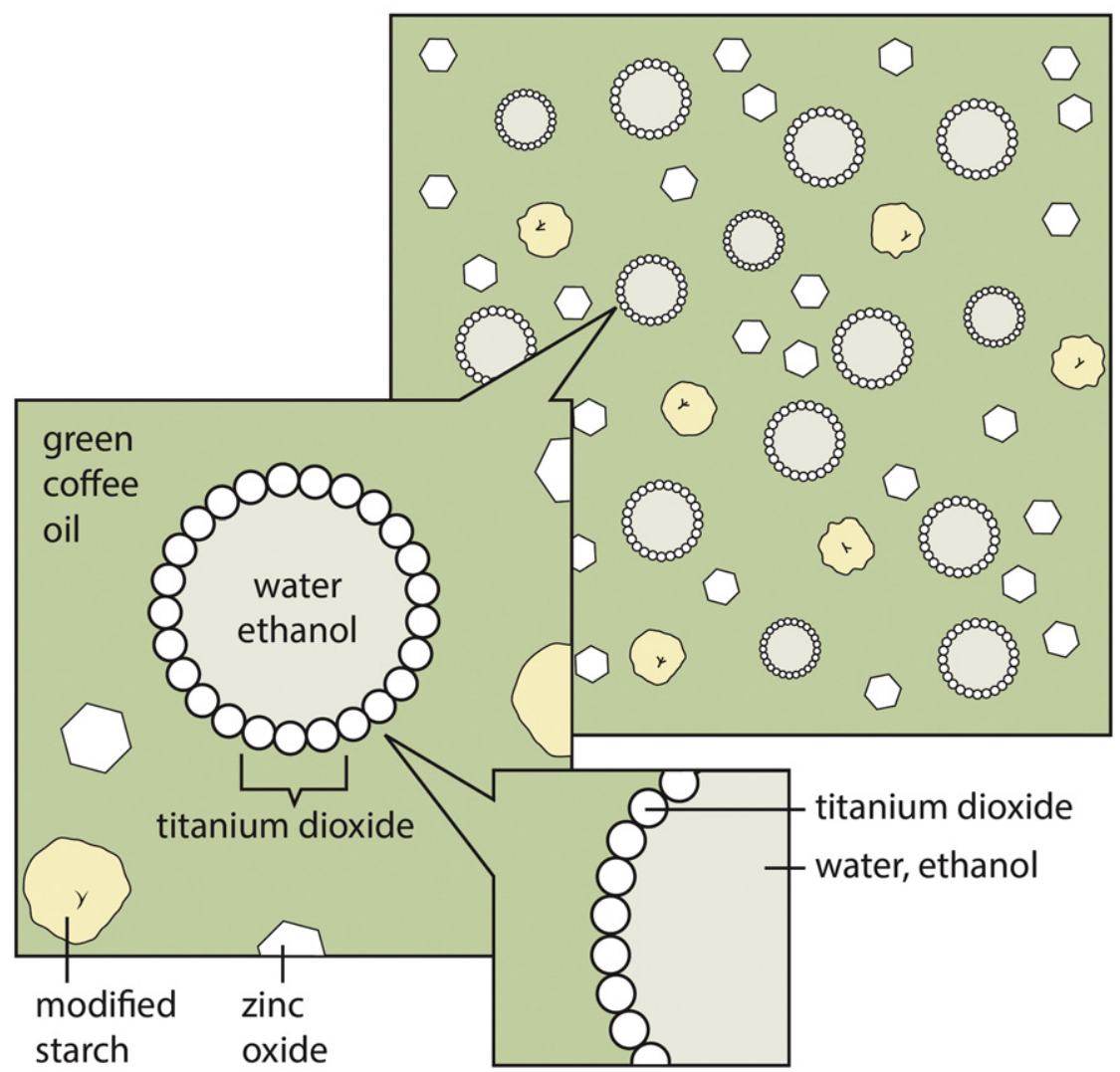

Fig. 2. Schematic representation of a water-in-oil Pickering emulsion (PS2) proposed by this research work. 
No reactions or skin sensitization/irritation were observed in the initial 3 weeks contact and even after the final challenge contact. Thus, very good skin compatibility was obtained for these sunscreen formulations.

3.3.6.1. In Vivo Sun Product Water Resistance. After ensuring the in vitro efficacy of these formulations, the WRR was also evaluated in humans. Human testing is considered to be the most acceptable and definitive method for claiming WRR. This method is a new in vivo screening approach to measure WRR using UVA-induced fluorescence imaging. Although it does not allow determining the exact SPF before and after the immersion, it evaluates the sunscreen loss due to the action of water. Similarly to the in vitro assay, the value for the $90 \%$ lower unilateral confidence limit must be mean \%WRR $\geq 50 \%$.

Skin whiteness showed a similar behaviour to that obtained for water resistance. The PS1 and PS2 emulsions possess 70-85\% of whiteness without water exposure. However, when exposed to water for $40 \mathrm{~min}$, both products showed quite similar amounts of whiteness on skin and quite perceptible, as shown in Fig. 3. Both emulsions presented a broad peak distribution ranging from 50 to $80 \%$ with a median peak around $70 \%$ of whiteness. Thus, w/o emulsions possess a higher degree of water repellency needed to avoid products to coalesce in contact with water drops remaining on wet skin.

Based on the WRR values (Table 4), it is possible to claim water resistance of the product. Furthermore, these in vivo values were higher than those obtained in vitro, which may be explained by a better adhesion to human skin compared with the adhesive tape used for the in vitro assay.

\section{Conclusions}

This work arose from the necessity to fill the gap in the photoprotection market whose attention is frequently more focused on cosmetically issues rather than maximum protection against both UVA and B radiation as well as cellular protection. Thus, a novel sunscreen formulation with a high UVA and B protection, biological activity and better tolerability was designed based on the Pickering emulsions concept. The successful formulation was possible by combining natural and multifunctional compounds. In particular, GCO (a recognized bioantioxidant) was associated to UV physical absorbers ( $\mathrm{TiO}_{2}$ and $\mathrm{ZnO}$ ) at lower concentrations and with rigorous particles size higher than $100 \mathrm{~nm}$. The UV filters concentration could be reduced due to the SPF improvement by the presence of both starch particles and GCO.

Pickering emulsions proved to be a promising solution for the sunscreen development and all results revealed an excellent compromise between stability, UV protection, rheological and mechanical behaviour, efficacy, safety and cosmeticity.

\section{Acknowledgments}

This work was supported by the Fundação para a Ciência e a Tecnologia, Portugal (UID/DTP/04138/2013 to iMed.ULisboa and grant SFRH/BDE/51599/2011) and Laboratórios Atral S.A., Portugal.

\section{References}

[1] S. Rangwala, K.Y. Tsai, Roles of the immune system in skin cancer, Br. J. Dermatol. 165 (2011) 953-965.
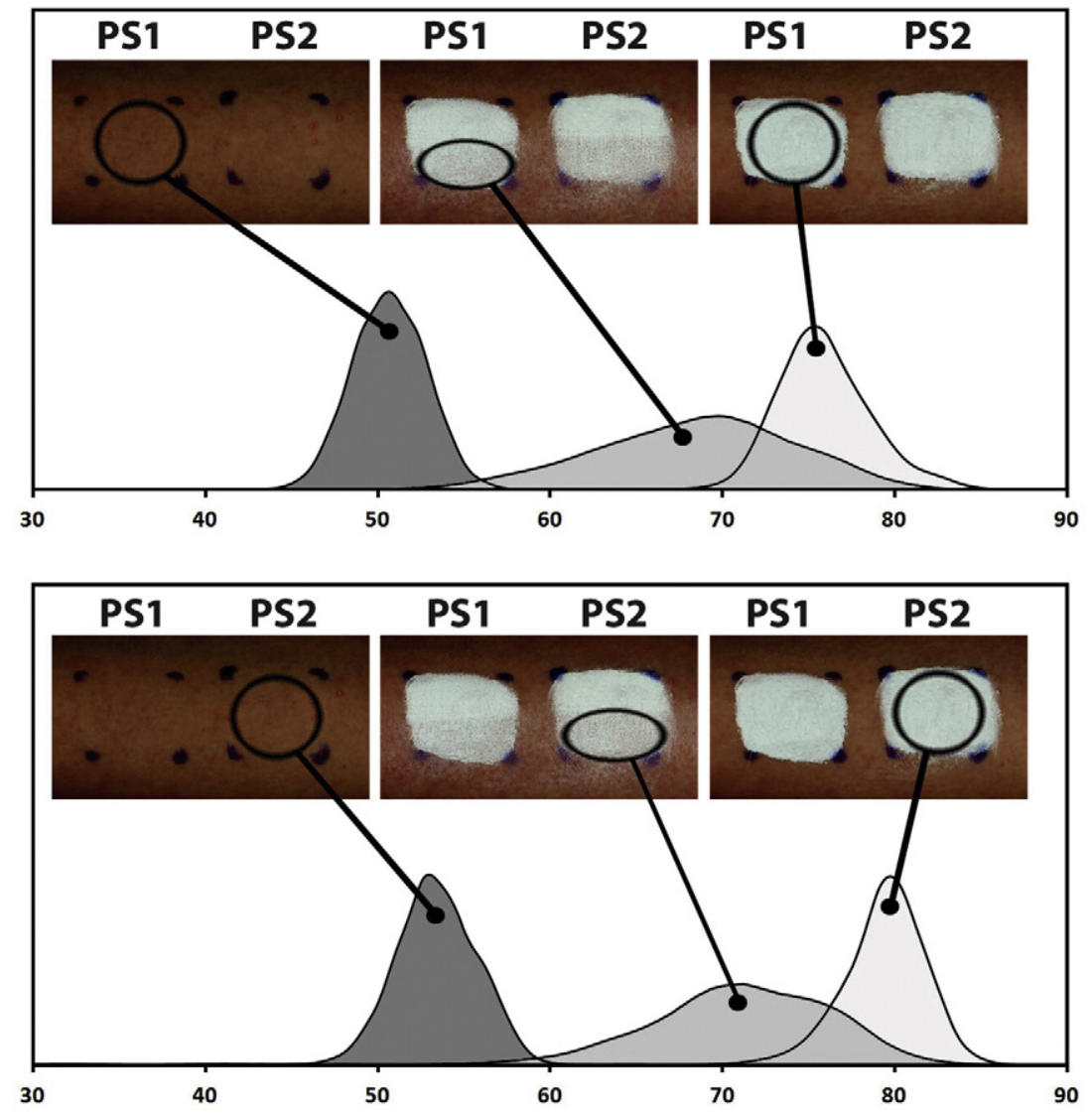

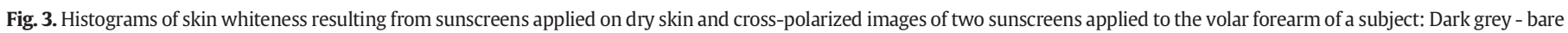
skin; light grey - fresh sunscreen applications with 30 min air drying; and grey - sunscreen after 40 min water immersion. 
[2] G.F.L. Hofbauer, J.N.B. Bavinck, S. Euvrard, Organ transplantation and skin cancer: basic problems and new perspectives, Exp. Dermatol. 19 (2010) 473-482.

[3] M. Yaar, B.A. Gilchrest, Ageing and photoageing of keratinocytes and melanocytes, Clin. Exp. Dermatol. 26 (2001) 583-591.

[4] I.M. Hadshiew, M.S. Eller, B.A. Gilchrest, Skin aging and photoaging: the role of DNA damage and repair, Clin. Exp. Dermatol. 11 (2000) 19-25.

[5] A. Ascenso, H. Ribeiro, H.C. Marques, H. Oliveira, C. Santos, S. Simões, Tretinoin still as a key agent for UV photoaging management, Mini-Rev. Med. Chem. 14 (2014) 629-641.

[6] E. Dutra, D. Oliveira, E. Kedor-Hackmann, M. Santoro, Determination of sun protection factor (SPF) of sunscreens by ultraviolet spectrophotometry, Braz. J. Pharm. Sci. 40 (2004) 381-385.

[7] A.K. Mishra, A. Mishra, P. Chattopadhyay, Evaluation of sun protection factor of some marketed formulations of sunscreens by ultraviolet spectroscopic method, J. Curr. Pharm. Res. 5 (2011) 32-35.

[8] F.P. Gasparro, M. Mitchnick, J.F. Nash, A review of sunscreen safety and efficacy, Photochem. Photobiol. 68 (1998) 243-256.

[9] J.S. Mansur, M.N.R. Breder, M.C.A. Mansur, R.D. Azulay, 24., Determinação do fator de proteção solar por espectrofotometria, An. Bras. Dermatol. 61 (1986) 167-172.

[10] F. Laredj-Bourezg, Y. Chevalier, O. Boyron, M.A. Bolzinger, Emulsions stabilized with organic solid particles, Colloids Surf. A Physicochem. Eng. Asp. 413 (2012) 252-259.

[11] J.W. Folter, M.W. Ruijven, K.P. Velikov, Oil-in-water Pickering emulsions stabilized by colloidal particles from the water-insoluble protein zein, Soft Matter 8 (2012) 6807-6815.

[12] J. Frelichowska, M.A. Bolzinger, J. Pelletier, J.P. Valour, Y. Chevalier, Topical delivery of lipophilic drugs from o/w Pickering emulsions, Int. J. Pharm. 371 (2009) 56-63.

[13] B. Nair, T.A. Yamarik, Final report on the safety assessment of aluminum starch octenylsuccinate, Int. J. Toxicol. 21 (Suppl. 1) (2002) 1-7.

[14] R.G. Holdich, I. Yilmaz, M. Lazrigh, G. Shama, Production and evaluation of floating photocatalytic composite particles formed using Pickering emulsions and membrane emulsification, Ind. Eng. Chem. Res. 51 (2012) 12509-12516.

[15] S.Q. Wang, R. Tooley, Photoprotection in the era of nanotechnology, Semin. Cutan. Med. Surg. J. 30 (2011) 210-213.

[16] T.G. Smijs, S. Pavel, Titanium dioxide and zinc oxide nanoparticles in sunscreens: focus on their safety and effectiveness, Nanotechnol. Sci. Appl. 12 (2011) 95-112.

[17] H. Ribeiro, J. Marto, S. Raposo, M. Agapito, V. Isaac, B. Chiari, P. Lisboa, A. Paiva, S. Barreiros, P. Simões, From coffee industry waste materials to skin-friendly products with improved skin fat levels, Eur. J. Lipid Sci. Technol. 115 (2013) 330-336.

[18] J. Marto, L. Gouveia, I.M. Jorge, A. Duarte, L.M. Gonçalves, S.M.C. Silva, F. Antunes, A.A.C.C. Pais, E. Oliveira, A.J. Almeida, H.M. Ribeiro, Starch-based Pickering emulsions for topical drug delivery: a QbD approach, Colloids Surf. B: Biointerfaces 135 (2015) 183-192.

[19] R.M. Sayre, P.P. Agin, G.J. Levee, E. Marlowe, Comparison of in vivo and in vitro testing of sunscreening formulas, Photochem. Photobiol. 29 (1979) 559-566.

[20] EC, in: EC (Ed.), Regulation (EC) No 1223/2009 of the European Parliament and of the Council of 30 November 2009 on Cosmetic Products, Official Journal of the European Union, 2009.

[21] S. Kale, P.P. Ghoge, A. Ansari, A. Waje, A. Sonawane, Formulation and in-vitro determination of sun protection factor of Nigella sativa Linn. seed oil sunscreen cream, Int. J. PharmTech Res. 2 (2010).

[22] COLIPA, Guidelines for Evaluating Sun Product Water Resistance, 2005.

[23] S. Ahn, H. Yang, H. Lee, S. Moon, I. Chang, Alternative evaluation method in vitro for the water-resistant effect of sunscreen products, Skin Res. Technol. 14 (2008) 187-191.
[24] BS, Methods for determination of particle size distribution, Part 4, Guide to Microscope and Image Analysis Methods, British Standards 3406-4:1993 1993, p. 36 .

[25] I. Standard, Microbiology - enumeration of yeast and mould, Microbiology Enumeration of Yeast and Mould, 2008.

[26] I. Standard, Microbiology - enumeration and detection of aerobic mesophilic bacteria, Microbiology - Enumeration and Detection of Aerobic Mesophilic Bacteria, 2006.

[27] I. Standard, Microbiology - general instructions for microbiological examination, Microbiology - General Instructions for Microbiological Examination, 2005.

[28] OECD, Test No. 439: In Vitro Skin Irritation Reconstructed Human Epidermis Test Method: Reconstructed Human Epidermis Test Method, OECD Publishing, 2010.

[29] F.N. Marzulli, H.I. Maibach, Contact allergy: predictive testing in man, Contact Dermatitis 2 (1976) 1-17.

[30] S. Fregert, H.J. Bandmann, International Contact Dermatitis Research Group Patch Testing, Springer-Verlag, New York, 1975 8-10.

[31] G. Singh, E.M. Joyce, J. Beddow, T.J. Mason, Evaluation of antibacterial activity of ZnO nanoparticles coated sonochemically onto textile fabrics, J. Microbiol. Biotechnol. Food Sci. 2 (2012) 106-120.

[32] Z.D. Draelos, Cosmetic Dermatology: Products and Procedures, Wiley, 2011.

[33] J. Marto, L. Gouveia, B. Chiari, A. Paiva, V. Isaac, P. Pinto, P. Simões, A. Almeida, H. Ribeiro, The green generation of sunscreens: using coffee industrial sub-products, Ind. Crop. Prod. 80 (2016) 93-100.

[34] J.N. Wintgens, Coffee: growing, processing, sustainable production, A Guidebook for Growers, Processors, Traders and Researchers, Wiley-Vch, 2009.

[35] S. Sasidharan, Y. Chen, D. Saravanan, K. Sundram, L.Y. Latha, Extraction, isolation and characterization of bioactive compounds from plants' extracts, Afr. J. Tradit. Complement. Altern. Med. 8 (2011).

[36] B.G. Chiari, E. Trovattib, E. Pecorarob, M.A. Corrêaa, R.M. Cicarellia, S. Ribeiro, V. Isaac, Synergistic effect of green coffee oil and synthetic sunscreen forhealth care application, Ind. Crop. Prod. 52 (2014) 389-393.

[37] J. Guth, G. Martino, J. Pasapane, D. Ronco, Polymeric approaches to skin protection, Cosmet. Toiletries 106 (1991) 71-74.

[38] N. Serpone, D. Dondi, A. Albini, Inorganic and organic UV filters: their role and efficacy in sunscreens and suncare products, Inorg. Chim. Acta 360 (2007) 794-802.

[39] M. Issavini, L. Ferrero, V. Alaro, U. Heinrich, H. Tronnier, D. Kockott, D. Lutz, V. ournier, M. Zambonin, M. Meloni, Determination of the in vitro SPF, Cosmet. Toiletries 118 (2003) 63-72.

[40] R.P. Stokes, D.L. Diffey, L.C. Dawson, S.P. Barton, A novel in vitro technique for measuring the water resistance of sunscreens, Int. J. Cosmet. Sci. 20 (1998) 235-340.

[41] R.P. Chhabra, J.F. Richardson, Non-newtonian fluid behaviour, in: R.P.C.F. Richardson (Ed.), Non-Newtonian Flow and Applied Rheology (Second Edition), ButterworthHeinemann, Oxford 2008, pp. 1-55.

[42] C.V. Nikiforidis, C.G. Biliaderis, V. Kiosseoglou, Rheological characteristics and physicochemical stability of dressing-type emulsions made of oil bodies-egg yolk blends, Food Chem. 134 (2012) 64-73.

[43] L.G. Torres, R. Iturbe, M.J. Snowden, B.Z. Chowdhry, S.A. Leharne, Preparation of o/w emulsions stabilized by solid particles and their characterization by oscillatory rheology, Colloids Surf. A Physicochem. Eng. Asp. 302 (2007) 439-448.

[44] C. Lin, D.D.L. Chung, Nanostructured fumed metal oxides for thermal interface pastes, J. Mater. Sci. 42 (2007) 10.

[45] S. Raposo, A. Salgado, L. Gonçalves, P. Pinto, M. Urbano, H. Ribeiro, Safety assessment and biological effects of a new cold processed SilEmulsion for dermatological purpose, BioMed Res. Int. 2013 (2013) 10. 